\title{
Polimorfismos en los genes de dihidrofolato-reductasa (dhfr) y dihidropteroato-sintasa (dhps) y modelado estructural del gen dhps en aislamientos colombianos de Toxoplasma gondii
}

\author{
Liliana Jazmín Cortés ${ }^{1}$, Sofía Duque ${ }^{1}$, Miryam Consuelo López², Diego Moncada³, Diego Molina³, \\ Jorge Enrique Gómez-Marín ${ }^{3}$, María Luz Gunturiz ${ }^{4}$ \\ 1 Grupo de Parasitología, Subdirección de Investigación Científica y Tecnológica, Instituto Nacional de Salud, \\ Bogotá D.C., Colombia \\ 2 Departamento de Parasitología, Facultad de Medicina, Universidad Nacional de Colombia, Bogotá D.C., Colombia \\ 3 Grupo de Parasitología Molecular (GEPAMOL), Centro de Investigaciones Biomédicas, Universidad del Quindío, \\ Armenia, Colombia \\ ${ }^{4}$ Grupo de Fisiología Molecular, Subdirección de Investigación Científica y Tecnológica, Instituto Nacional de \\ Salud, Bogotá D.C., Colombia
}

Lugares donde se realizó la investigación:

Grupo de Parasitología y Grupo de Fisiología Molecular, Subdirección de Investigación Científica y Tecnológica, Instituto Nacional de Salud, Bogotá, D.C., Colombia, y Centro de Investigaciones Biomédicas, Universidad del Quindío, Armenia, Colombia.

Introducción. No existen reportes sobre las variaciones en la secuencia de los genes blanco de los medicamentos anti-Toxoplasma en aislamientos provenientes de Suramérica.

Objetivo. Clonar y secuenciar los genes de la dihidrofolato-reductasa ( $d$ hfr) y la dihidropteroato-sintetasa (dhps) de la cepa de referencia $\mathrm{RH}$ y de dos aislamientos colombianos de Toxoplasma gondii.

Materiales y métodos. Se obtuvieron dos aislamientos de $T$. gondii en líquido céfalorraquídeo de pacientes colombianos positivos para HIV con toxoplasmosis cerebral. Se extrajo el ADN de los genes dhfr y dhps y se amplificaron mediante reacción en cadena de la polimerasa (PCR). Los productos fueron clonados en el vector PGEM-T y secuenciados.

Resultados. Se encontró un cambio de adenina por guanina $(A \leftrightarrow G)$ en la posición 235 del exón 2 del gen dhps, dos cambios de guanina por citocina $(G \leftrightarrow C)$ en las posiciones 259 y 260 y un cambio de timina por guanina $(T \leftrightarrow G)$ en la posición 371 del exón 4 del gen dhps. Por análisis bioinformático, en este último exón se identificó un polimorfismo no sinónimo en la región codificante, que podría llevar al cambio de una Glu (CAA o CAG) por una His (codificada por los codones AAU o AAC). Se calculó el modelo estructural de la enzima dihidropteroato-sintetasa (DHPS) de $T$. gondii y se identificaron las modificaciones en la estructura secundaria ocasionadas por las mutaciones.

Conclusiones. La metodología estandarizada puede servir como base para la búsqueda de polimorfismos en muestras de pacientes con diferentes manifestaciones clínicas de toxoplasmosis y para establecer su posible relación con los cambios en la sensibilidad a los antifolatos y la reacción al tratamiento.

Palabras clave: Toxoplasma, reacción en cadena de la polimerasa, resistencia a medicamentos, ácido fólico/antagonistas e inhibidores, conformación molecular.

doi: http://dx.doi.org/10.7705/biomedica.v34i4.2132

Gene polymorphisms in the dihydrofolate reductase (dhfr) and dihydropteroate synthase (dhps) genes and structural modelling of the dhps gene in Colombian isolates of Toxoplasma gondii

Introduction: There are no reports describing polymorphisms in target genes of anti-Toxoplasma drugs in South American isolates.

Objective: This study sought to perform cloning and sequencing of the dihydrofolate reductase (dhfr) and dihydropteroate-synthase (dhps) genes of the reference Rh strain and two Colombian isolates of Toxoplasma gondii.

\footnotetext{
Contribución de los autores: Liliana Jazmín Cortés: realización de los experimentos

Sofía Duque Beltrán: análisis de los resultados

Diego Moncada y Diego Molina: análisis bioinformático y modelado estructural

María Luz Gunturiz: realización de experimentos, análisis de resultados y análisis bioinformático

Todos los autores participaron en la redacción del artículo.
}

Liliana Jazmín Cortés, Myriam Consuelo López y Jorge Enrique Gómez-Marín: diseño del proyecto y análisis de resultados 
Materials and methods: Two isolates were obtained from the cerebrospinal fluid of HIV-infected patients with cerebral toxoplasmosis. A DNA extraction technique and PCR assay for the dhfr and dhps genes were standardized, and the products of amplification were cloned into Escherichia coli and sequenced.

Results: One polymorphism $(A \leftrightarrow G)$ was found at position 235 of exon 2 in the dhps gene. In addition, two polymorphisms $(\mathrm{G} \leftrightarrow \mathrm{C})$ at positions 259 and 260 and one polymorphism $(\mathrm{T} \leftrightarrow \mathrm{G})$ at position 371 within exon 4 of the dhps gene were detected. In this last exon, a bioinformatic analysis revealed a non-synonymous polymorphism in the coding region that could lead to the substitution of Glu (CAA or CAG) for His (encoded by codons AAU or AAC). A structural model of the T. gondii DHPS protein was calculated, and the results revealed modifications in secondary structure due to mutations.

Conclusions: The methods described in this study can be used as a tool to search for polymorphisms in samples from patients with different clinical manifestations of toxoplasmosis and to examine their relationship with the therapeutic response.

Key words: Toxoplasma, polymerase chain reaction, drug resistance, folic acid/antagonists and inhibitors, molecular conformation.

doi: http://dx.doi.org/10.7705/biomedica.v34i4.2132

La toxoplasmosis es una zoonosis que frecuentemente cursa como una infección asintomática en los humanos. Sin embargo, se pueden presentar diferentes manifestaciones clínicas de la enfermedad en recién nacidos, en niños en sus primeros años de edad y en pacientes inmunosuprimidos (1-4).

La combinación sinérgica de pirimetamina y sulfadoxina, que inhibe los pasos secuenciales en la síntesis del ácido fólico, es el tratamiento más utilizado para la toxoplasmosis (5). Se han documentado fracasos terapéuticos relacionados con factores del huésped, como la intolerancia al medicamento o la mala absorción, o del parásito, como el desarrollo de resistencia a los medicamentos o la baja sensibilidad de algunas cepas de Toxoplasma al tratamiento $(6,7)$. Se han encontrado lesiones oculares hasta en el $21 \%$ de pacientes con coriorretinitis y en niños con toxoplasmosis congénita, a pesar de haber recibido tratamiento adecuado durante un año $(8,9)$.

En Colombia existen algunos reportes sobre la ineficacia del tratamiento (sulfadoxina y pirimetamina más clindamicina) en pacientes con toxoplasmosis cerebral, sin que se hayan establecido las causas del fracaso terapéutico (10). Aunque no se ha comprobado la resistencia de Toxoplasma gondii a los antifolatos, en algunos estudios se ha revelado la existencia de cepas con algún tipo de resistencia

\footnotetext{
Correspondencia:

Liliana Jazmín Cortés, Grupo de Parasitología, Subdirección de Investigación Científica y Tecnológica, Instituto Nacional de Salud, Avenida calle 26 N 51-20, Zona 6, CAN, Bogotá, D.C., Colombia

Teléfono: (571) 2207700 , extensión 1337

jcortes@ins.gov.co

Recibido: 16/09/13; aceptado: 09/06/14
}

a la pirimetamina y a la sulfadoxina (in vivo e in vitro), e incluso, se han hecho algunas aproximaciones en proteómica en cepas resistentes aisladas de pacientes $(6,11-14)$. Una concentración de antifolatos inferior a la dosis terapéutica de mantenimiento en pacientes inmunosuprimidos $(500 \mathrm{mg}$ de sulfadoxina, cuatro veces al día, y $25 \mathrm{mg}$ diarios de pirimetamina) (15), o los tratamientos prolongados que son necesarios en esta infección parasitaria (16), pueden contribuir al fracaso clínico debido a la aparición de variantes resistentes del parásito, las cuales pueden cambiar sus estrategias de supervivencia, inhibiendo la respuesta a los medicamentos, expulsando rápidamente el medicamento de sus células o induciendo cambios en la estructura de las enzimas dihidrofolato-reductasa y dihidropteroato-sintetasa para inhibir la acción de los antifolatos $(13,17)$.

Respecto a las mutaciones del gen dhfr que confieren resistencia a las diamino-pirimidinas, este fenómeno se debe en gran medida a la presión ejercida por los medicamentos, lo que ha permitido la selección de una serie de mutaciones sucesivas en los genes $d h f r$ y dhps. En el caso de T. gondii, se han reportado algunas cepas resistentes a la pirimetamina (18). Sin embargo, el gen dhfr no ha demostrado variación en la secuencia con respecto a la cepa silvestre y actualmente no existen datos concluyentes sobre la base molecular de la resistencia a la pirimetamina en este parásito, con excepción de la demostración experimental de que las mutaciones dirigidas confieren resistencia a este medicamento (19).

La mutación más frecuente en los genes que codifican para la enzima dihidrofolato-reductasa se presenta en el codón 156. La mutación en el gen dhfr cambia CTG por TTG (ambos codifican 
para leucina), es silenciosa, sin que hasta ahora haya sido relacionada con efectos biológicos, y solo se ha encontrado en algunos aislamientos del genotipo $\mathrm{C}$ dhps, el cual es una variante alélica del parásito que presenta una variación específica en cuatro de los cinco loci polimorfos del gen dhps y afecta los codones 474, 560, 580, 597 y 627 (13).

Las mutaciones más frecuentes en los genes que codifican para la enzima dihidropteroato-sintetasa se presentan en los codones 407, 474, 560 y 597 (13).

En Colombia se sabe de recaídas de la infección en pacientes que han recibido tratamiento completo, pero no se conocen las causas $(10,20)$, por lo que es relevante estudiar el polimorfismo genético de los aislamientos del parásito. El hallazgo de mutaciones en los codones 407, 474, 560 y 597 del gen dhps o en el codón 156 del gen dhfr de aislamientos de $T$. gondii obtenidos de pacientes con toxoplasmosis, podría relacionarse con cambios en la sensibilidad o resistencia del parásito a la pirimetamina y la sulfadoxina, medicamentos utilizados en el tratamiento de esta parasitosis, lo que podría explicar estas recaídas.

En estudios previos en Colombia se han reportado polimorfismos en genes homólogos de Plasmodium spp., pero no existen reportes para $T$. gondii (21-24). Este estudio tuvo como objetivo hacer la clonación y la secuenciación de los genes dihidrofolato-reductasa ( $d h f r$ ) y dihidropteroatosintetasa (dhps) de la cepa de referencia $\mathrm{RH}$ y de dos aislamientos de T. gondii obtenidos a partir del líquido cefalorraquídeo de pacientes positivos para HIV con toxoplasmosis cerebral. La descripción de estos polimorfismos en cepas colombianas aporta conocimiento de base para la generación de nuevas líneas de investigación encaminadas a determinar la relación de estas mutaciones con una respuesta inadecuada al tratamiento, lo que a su vez pudiera explicar la presencia de recaídas, especialmente en pacientes con toxoplasmosis ocular y congénita.

\section{Materiales y métodos}

\section{Muestras biológicas}

Se obtuvo líquido cefalorraquídeo de dos pacientes positivos para el virus de la inmunodeficiencia humana (HIV) con toxoplasmosis cerebral, previo consentimiento informado de los pacientes y su autorización para usar muestras biológicas en estudios posteriores, cuya prueba serológica de inmunofluorescencia indirecta fue positiva (el resultado se considera positivo a partir de 1/16), con títulos de anticuerpos IgG frente a $T$. gondii de $1 / 2.048$ y $1 / 4.096$, respectivamente.

\section{Aislamiento de Toxoplasma gondii a partir de líquido cefalorraquídeo}

Por vía intraperitoneal se inocularon $0,4 \mathrm{ml}$ del sedimento de líquido cefalorraquídeo de dos pacientes positivos para HIV y con toxoplasmosis cerebral confirmada, en ratones machos, cepa ICR-CD1, de 20 días de edad, procedentes del Bioterio del Instituto Nacional de Salud (dos ratones por cada muestra y un ratón control sin inocular por cada jaula), con el fin de evaluar en forma sucesiva el comportamiento del modelo animal sin inoculación, bajo las mismas condiciones de los ratones inoculados con los exudados que contenían el parásito. Durante el experimento se controlaron las variables de sintomatología de la infección por toxoplasma en los ratones: piloerección, hepato-esplenomegalia y letargia, además de la presencia o ausencia de taquizoítos en el exudado peritoneal (25).

Los ratones inoculados se observaron durante dos semanas, tiempo en el cual no presentaron ninguna sintomatología indicativa de infección por toxoplasma. Al cabo de este tiempo, los ratones se sacrificaron en cámara de $\mathrm{CO}_{2}$, se hizo extracción de exudado peritoneal ( $2 \mathrm{ml}$ por cada ratón) y pase en ciego a otros seis ratones. A partir de este material se hicieron cinco pases sucesivos (seis ratones por pase) hasta obtener una reacción positiva del exudado peritoneal con manifestaciones clínicas indicativas de toxoplasmosis en los ratones $(26,27)$.

\section{Extracción de ADN y técnica de reacción en cadena de la polimerasa}

El ADN se extrajo empleando dos metodologías: el estuche comercial PureLinkTM® y el método de lisis alcalina estandarizado en el Laboratorio de Fisiología Molecular del Instituto Nacional de Salud.

Los análisis moleculares se hicieron con ADN genómico del parásito extraído de las dos muestras de pacientes y de la cepa de referencia $\mathrm{RH}$ de $T$. gondii. Para ello, se emplearon los oligonucleótidos reportados por Aspinall, et al. (13), y se optimizaron las condiciones de amplificación para cada uno de los fragmentos de los genes dhfry dhps, de acuerdo con las temperaturas óptimas de hibridación de los oligonucleótidos y el tamaño del fragmento esperado y, en lo concerniente a los tiempos de desnaturalización y de extensión, el número de ciclos y el tiempo del ciclo de extensión final. 
La mezcla de reacción para la amplificación de ambos genes se optimizó así: solución tampón, 1X; oligonucleótidos directo e inverso, $500 \mu \mathrm{m}$; DNTP, $200 \mu \mathrm{m} ; \mathrm{MgCl}_{2}$ en concentración de $150 \mu \mathrm{m} ; 1 \mathrm{U}$ Taq, y $4 \mu \mathrm{l}$ de ADN, para un volumen final de $50 \mu \mathrm{l}$. Las condiciones de amplificación se encuentran en el cuadro 1.

El producto de amplificación se visualizó mediante una electroforesis en gel de agarosa (1,8\%), con solución tampón TBE $1 \mathrm{X}$ y bromuro de etidio en una concentración de $0,5 \mu \mathrm{g} / \mathrm{ml}$. Una vez obtenidos los productos de amplificación, se purificaron a partir de gel empleando el estuche llustra GFXPCR ADN and Gel Band Purification (GE Healthcare), según las indicaciones del fabricante.

\section{Clonación y secuenciación}

Con los productos purificados se hizo la secuenciación directa por el método de Sanger en el Grupo de Fisiología Molecular del Instituto Nacional de Salud, empleando los oligonucleótidos de PCR para cada uno de los fragmentos. Se clonaron los productos purificados verificados por secuenciación utilizando el vector pGEM-T y células 'termocompetentes' de Escherichia coli JM109 (Promega). Los clones recombinantes para cada uno de los exones se seleccionaron usando como agente selectivo la ampicilina y se verificaron mediante PCR. A partir del ADN de los plásmidos de, por lo menos, tres clones por exón, se secuenciaron los ácidos nucleicos empleando el método de Sanger y el analizador genético ABI Prism 310.

\section{Análisis bioinformático y modelado estructural in silico}

Las secuencias obtenidas se editaron y se generó una secuencia de consenso por cada clon. Se hizo un alineamiento mediante Clustal W y se comparó con las secuencias reportadas en las bases de datos del National Center for Biotechnology Information (NCBI: http://blast.ncbi.nlm.nih.gov/
Blast. cgi). Se diseñó un modelo estructural por homología de la proteína dihidropteroato-sintetasa TGME49_259550 de T. gondii ME49 con el servidor ITASSER (http://zhanglab.ccmb.med.umich. edu/l-TASSER/). Las regiones funcionales de las secuencias se detectaron con la herramienta en línea SMART (http://smart.embl-heidelberg.de/) y con ProFunc (http://www.ebi.ac.uk/thornton-srv/ databases/ProFunc/). El análisis y la visualización de la estructura se llevaron a cabo en el programa Chimera 1.8.5 ( http://www.cgl.ucsf.edu/chimera/).

\section{Resultados}

Obtención de los aislamientos colombianos de Toxoplasma gondii a partir de líquido cefalorraquídeo

Se hicieron cinco pases ciegos sucesivos durante 43 días, al cabo de los cuales los dos ratones de control continuaban sin presentar ninguna sintomatología; los cuatro ratones inoculados con el líquido cefalorraquídeo de los pacientes, presentaban piloerección, hepato-esplenomegalia y letargia. Excepto en los animales de control, en el exudado peritoneal de los ratones sacrificados se observaron taquizoítos de $T$. gondii, hallazgo que fue confirmado al examinar los extendidos coloreados con la tinción de Romanowsky modificada.

\section{Amplificación por PCR de los exones de los genes dhfr y dhps}

Mediante los dos métodos de extracción (lisis alcalina y estuche comercial) se obtuvo ADN de buena calidad, el cual fue verificado por electroforesis en gel de agarosa al $1 \%$ (banda $>10 \mathrm{~kb}$ ) y por espectrofotometría a $260 \mathrm{~nm}$ (relación 260/280 entre 1,8 y 2,0). Las condiciones para la amplificación por PCR de los fragmentos de los diferentes exones se describen en los cuadros 1 y 2 .

La amplificación por PCR de los exones 1, 2, 4 y 5 del gen dhps y del exón 2 del gen dhfr, permitió obtener una banda de 470 pb para el exón 1, una

Cuadro 1. Condiciones de las PCR para polimorfismos en los genes dhfr y dhps

\begin{tabular}{|c|c|c|c|c|c|}
\hline $\begin{array}{l}\text { Exones/ } \\
\text { condiciones }\end{array}$ & $\begin{array}{c}\text { dhps/exón } 1 \\
\text { Fragmento } \\
\text { esperado: } 470 \text { pb }\end{array}$ & $\begin{array}{c}\text { dhps/exón } 2 \\
\text { Fragmento } \\
\text { esperado: } 575 \text { pb }\end{array}$ & $\begin{array}{c}\text { dhps/exón } 4 \\
\text { Fragmento } \\
\text { esperado: } 622 \text { pb }\end{array}$ & $\begin{array}{c}\text { dhps/exón } 5 \\
\text { Fragmento } \\
\text { esperado: } 457 \mathrm{pb}\end{array}$ & $\begin{array}{c}\text { dhfr/exón } 2 \\
\text { Fragmento } \\
\text { esperado: } 379 \text { pb }\end{array}$ \\
\hline & $94^{\circ} \mathrm{C} / 5$ minutos & $94^{\circ} \mathrm{C} / 5$ minutos & $94^{\circ} \mathrm{C} / 5$ minutos & $94^{\circ} \mathrm{C} / 5$ minutos & $94^{\circ} \mathrm{C} / 5$ minutos \\
\hline Ciclos & 35 ciclos & 35 ciclos & 35 ciclos & 35 ciclos & 40 ciclos \\
\hline Desnaturalización & $94^{\circ} \mathrm{C} / 1$ minuto & $94^{\circ} \mathrm{C} / 1$ minuto & $94^{\circ} \mathrm{C} / 1$ minuto & $94^{\circ} \mathrm{C} / 1$ minuto & $94^{\circ} \mathrm{C} / 1$ minuto \\
\hline Anillamiento & $59,5^{\circ} \mathrm{C} / 1$ minuto & $59,1^{\circ} \mathrm{C} / 1$ minuto & $58,5^{\circ} \mathrm{C} / 1$ minuto & $59^{\circ} \mathrm{C} / 1$ minuto & $60^{\circ} \mathrm{C} / 1$ minuto \\
\hline Extensión & $72^{\circ} \mathrm{C} / 1$ minuto & $72{ }^{\circ} \mathrm{C} / 1$ minuto & $72^{\circ} \mathrm{C} / 1$ minuto & $72^{\circ} \mathrm{C} / 1$ minuto & $72^{\circ} \mathrm{C} / 1$ minuto \\
\hline Extensión final & $72^{\circ} \mathrm{C} / 7$ minutos & $72^{\circ} \mathrm{C} / 7$ minutos & $72^{\circ} \mathrm{C} / 7$ minutos & $72^{\circ} \mathrm{C} / 7$ minutos & $72^{\circ} \mathrm{C} / 7$ minutos \\
\hline
\end{tabular}

dhps: gen de la dihidropteroato-sintetasa; $d h f r$ gen de la dihidrofolato-reductasa 
Cuadro 2. Secuencias de los oligonucleótidos empleados

\begin{tabular}{|c|c|}
\hline $\begin{array}{l}\text { Nombre del } \\
\text { fragmento por } \\
\text { amplificar }\end{array}$ & PCR de oligonucleótidos \\
\hline \multirow[t]{3}{*}{ dhps exón 1ab } & Directo 5-GCGGTGTTTCGCAGCGACGACTG-3 \\
\hline & Reverso 5-CCTTCATGGTGTCAACGGAAATG-3 \\
\hline & Fragmento esperado: $470 \mathrm{pb}$ \\
\hline \multirow[t]{3}{*}{ dhps exón 2} & Directo 5-GGATGAAGTATAGATACATCTGC-3 \\
\hline & Reverso 5-CGGGTATTGCATGTTCTTTCACG-3 \\
\hline & Fragmento esperado: $575 \mathrm{pb}$ \\
\hline \multirow[t]{3}{*}{ dhps exón 4} & Directo 5-CAGTTGAACGTCACTTCCATATG-3 \\
\hline & Reverso 5-GCCAGCACACTGGAAGTGAAAC-3 \\
\hline & Fragmento esperado: 622pb \\
\hline \multirow[t]{3}{*}{ dhps exón 5} & Directo 5-GTGAAGTGATTGTGACCTGTCGCTAGC-3 \\
\hline & Reverso 5-CCGATCTACAGTCACGACGAAGG-3 \\
\hline & Fragmento esperado: $457 \mathrm{pb}$ \\
\hline \multirow[t]{3}{*}{ dhfr exón 2} & Directo 5-CGATGCAACTATAGGTTTCCTGG-3 \\
\hline & Reverso 5-TCCTTGCCAGTCTTCCCTGTATC-3 \\
\hline & Fragmento esperado: $379 \mathrm{pb}$ \\
\hline
\end{tabular}

dhps: gen de la dihidropteroato-sintetasa; dhfr: gen de la dihidrofolatoreductasa

de 575 pb para el exón 2, una de 622 pb para el exón 4 y una de 457 pb para el exón 5 del gen dhps, y una de 379 para el exón 2 del gen $d h f r$ (figuras 1-3). En el gel de agarosa al 1,8\% se visualizaron las bandas esperadas para cada exón.

Las secuencias obtenidas de los productos de amplificación y de los diferentes clones recombinantes, se compararon con las disponibles en Refseq (NCBI) y se observó que las secuencias obtenidas para los exones 1 y 5 del gen dhps y del exón 2 del gen dhfr eran idénticas a las reportadas

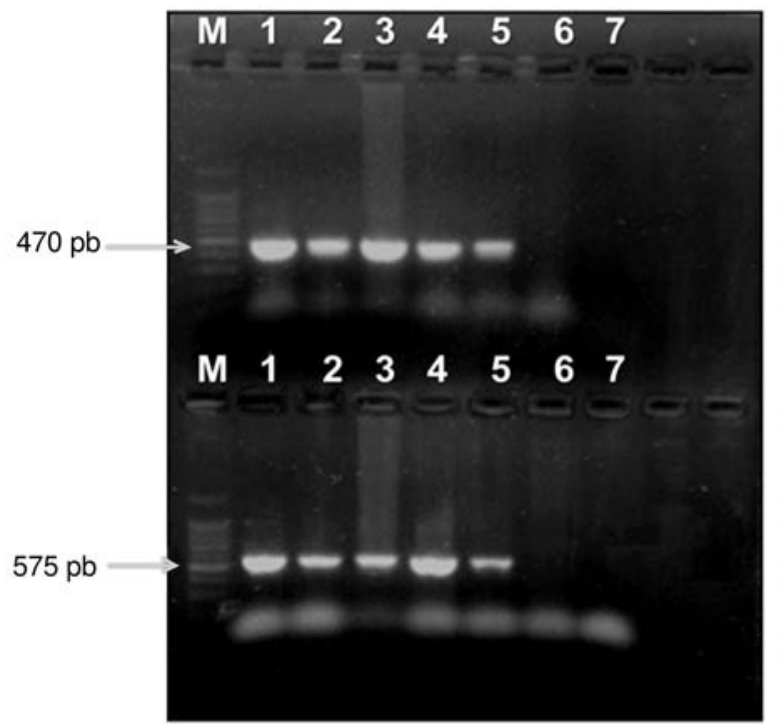

en la base de datos de ToxoDB (http://toxodb. org). Sin embargo, se encontró un cambio en un nucleótido de las tres muestras secuenciadas para el exón 2 del gen dhps en la posición 235 (figuras 4 y 5) y, al menos, tres cambios para el exón 4 del gen dhps a nivel de nucleótidos (posiciones 259-260 y 371). Estos polimorfismos llevaron a un cambio no sinónimo de una glutamina (CAA o CAG) por una histidina (codificada por los codones AAU o AAC) en la región codificante (figura 6).

\section{Modelado de la enzima dihidropteroato-sintetasa con polimorfismos $y$ sin ellos}

El modelo estructural obtenido de la enzima dihidropteroato-sintetasa sin mutación obtuvo un puntaje ( $C$ score) de 0,90 , presentando un plegamiento de tipo barril triosa-fosfato-isomerasa, con ocho hélices alfa en derredor de un barril central compuesto por ocho láminas beta (figura 7). Las hélices (1-8) se ubicaron entre los aminoácidos 38-53,74-93,105-115,134-143,166-185, 203-218, 234-242, 265-276, 285-303 y, las láminas del barril (1-8), entre los residuos 19-26, 55-61, 98-102, 118-122, 145-150, 191-195, 251-255 y 280-283. La estructura mutada presentaba una histidina (His) en la hélice alfa 6 en lugar de la glutamina (Glu). En el modelo se encontró que en la proteína mutada se ubicaban dos estructuras secundarias adicionales, una de las cuales correspondía una horquilla de láminas beta con los residuos insertados entre las posiciones 251 y 263, junto a la séptima y octava hélices coloreadas en magenta (figura 7).

Amplificación del producto del gen dhps-exón 1

M: Marcador de peso molecular de 100 pb-Promega

Carril 1: Muestra NR (Extracción con estuche comercial)

Carril 2: Muestra FA (Extracción con estuche comercial)

Carril 3: Muestra RH (Extracción con estuche comercial)

Carril 4: Muestra NR (Extracción manual)

Carril 5: Muestra FA (Extracción manual)

Carril 6: Muestra RH (Extracción manual)

Carril 7: Control negativo PCR

Amplificación del producto del gen dhps-exón 2

M: Marcador de peso molecular de 100 pb-Promega

Carril 1: Muestra NR (Extracción con estuche comercial)

Carril 2: Muestra FA (Extracción con estuche comercial)

Carril 3: Muestra RH (Extracción con estuche comercial)

Carril 4: Muestra NR (Extracción manual)

Carril 5: Muestra FA (Extracción manual)

Carril 6: Muestra RH (Extracción manual)

Carril 7: Control negativo PCR

Figura 1. Amplificación de los productos de los exones 1 y 2 del gen dhps en gel de agarosa al 1,8\%. En el carril 1 (M) se muestra el marcador de tamaño molecular de 100 pb (Promega); las flechas señalan las bandas de amplificación de los productos esperados, que corresponden a $470 \mathrm{pb}$ y $575 \mathrm{pb}$. 


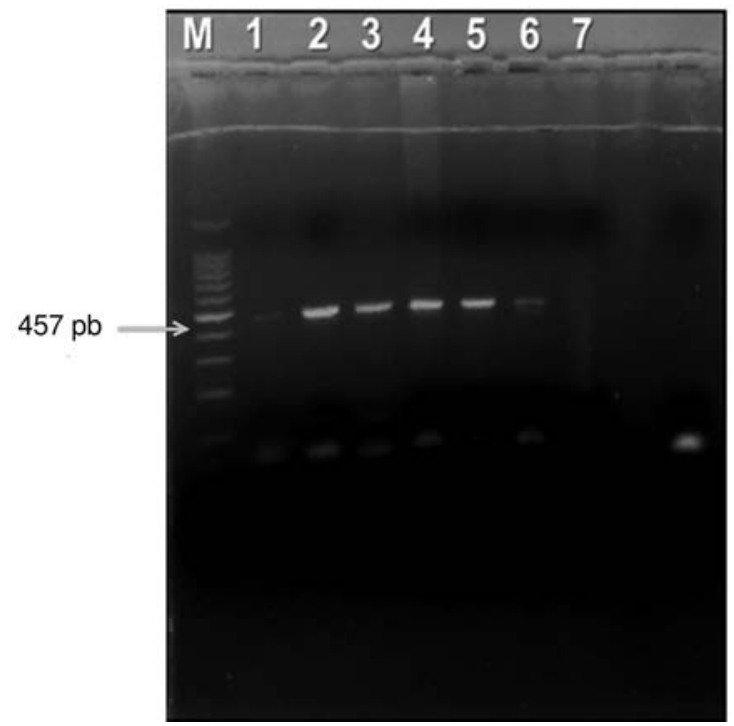

Amplificación del producto del gen dhps-exón 5

M: Marcador de peso molecular de 100 pb-Promega

Carril 1: Muestra NR (Extracción con estuche comercial)

Carril 2: Muestra FA (Extracción con estuche comercial)

Carril 3: Muestra RH (Extracción con estuche comercial)

Carril 4: Muestra NR (Extracción manual)

Carril 5: Muestra FA (Extracción manual)

Carril 6: Muestra RH (Extracción manual)

Carril 7: Control negativo PCR

Figura 2. Amplificación del producto del exón 5 del gen dhps en gel de agarosa al 1,8\%. En el carril 1 (M) se muestra el marcador de tamaño molecular de 100 pb (Promega); la flecha señala la banda de amplificación del producto esperado, que corresponde a $457 \mathrm{pb}$.

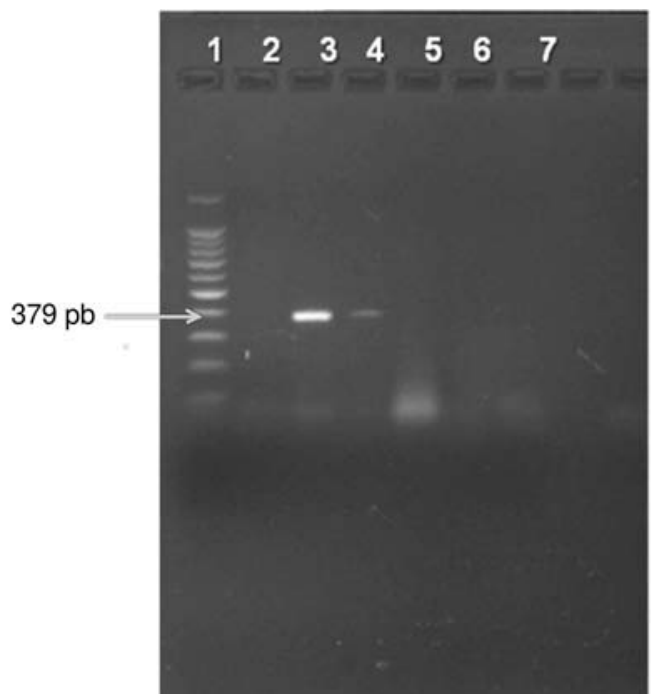

Amplificación del producto del gen dhfrexón 2

M: Marcador de peso molecular de $100 \mathrm{pb}$-Promega

Carril 1: Muestra NR (Extracción con estuche comercial)

Carril 2: Muestra FA (Extracción con estuche comercial)

Carril 3: Muestra RH (Extracción con estuche comercial)

Carril 4: Muestra NR (Extracción manual)

Carril 5: Muestra FA (Extracción manual)

Carril 6: Muestra RH (Extracción manual)

Carril 7: Control negativo PCR

Figura 3. Amplificación del producto del exón 2 del gen dhfr en gel de agarosa al 1,8\%. En el carril 1 (M) se muestra el marcador de tamaño molecular de 100 pb (Promega); la flecha señala la banda de amplificación del producto esperado, que corresponde a 379 pb.

\section{Discusión}

La técnica de PCR descrita para la detección de polimorfismos en los genes dhfr y dhps, así como las técnicas para su secuenciación y clonación, pueden utilizarse para futuras búsquedas de polimorfismos en diferentes aislamientos de $T$. gondii provenientes de distintas regiones del país (28), lo cual podría llevarse a cabo en un laboratorio de referencia como el Laboratorio Nacional de Referencia en Parasitología del Instituto Nacional de Salud, en tanto que la técnica de PCR podría implementarse en los laboratorios de salud pública de Colombia que cuenten con la infraestructura para ello.

En el presente trabajo se encontró el cambio de un nucleótido en las tres muestras secuenciadas para el exón 2 del gen dhps (posición 235), y para el exón 4 del gen dhps se encontraron, al menos, tres cambios (posiciones 259, 260 y 371). La mutación más frecuente en los genes que codifican para la enzima dihidrofolato-reductasa se presenta en el codón 156 (exón 2). La mutación en el gen dhfr, 

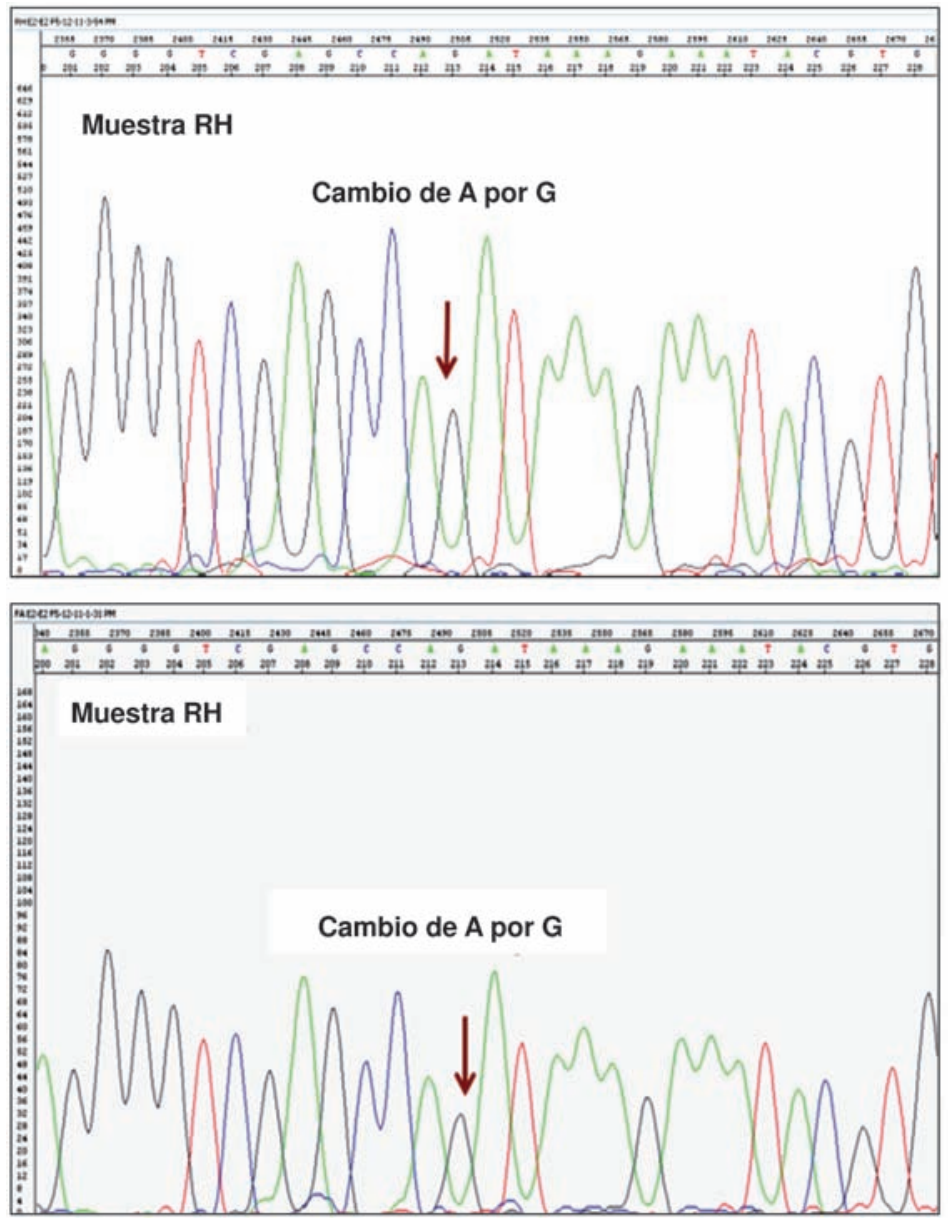

Figura 4. Electroferograma de fragmento de secuencia en el exón 2 del dhps obtenida para las muestras de dos pacientes empleando el oligonucleótido directo. La flecha indica el cambio encontrado con respecto a la secuencia reportada en las bases de datos.

Muestra RH: cambios con relación a la secuencia de la cepa de referencia RH (cepa ATCC 50174) que cambia CTG por TTG (ambos codifican para leucina), es una mutación silenciosa que hasta ahora no ha sido relacionada con ningún efecto biológico y solo se ha encontrado en algunos aislamientos de T. gondii del genotipo C dhps (13). No se encontraron polimorfismos en este exón para la cepa $\mathrm{RH}$ ni para las cepas de $T$. gondii de los pacientes. Sin embargo, la presencia o ausencia de este cambio de nucleótidos debería buscarse en una cantidad mucho mayor de muestras de pacientes con diferentes manifestaciones clínicas de toxoplasmosis.

En cuanto a la enzima dihidropteroato-sintetasa, las mutaciones más frecuentes en los genes que codifican para esta enzima se presentaron en los codones 407 (exón 1), 474 (exón 2), 560 (exón 4) y 597 (exón 5). En el presente trabajo se observó un polimorfismo en el exón 2 del gen dhps (posición 235) que llevó a un polimorfismo Ts $(A \leftrightarrow G)$; respecto a la mutación 560 (exón 4) reportada por Aspinall, et al. (13), como un cambio de arginina (AGG) por lisina (AAG), se identificó una mutación en el exón 4 mediante ensayos de bioinformática, así como dos cambios $(\mathrm{G} \leftrightarrow \mathrm{C})$ y un cambio $(\mathrm{T} \leftrightarrow \mathrm{G})$ no sinónimos en la región codificante, que llevaron al cambio de una Glu (CAA o CAG) por una His (codificada por los codones AAU o AAC).

De acuerdo con la predicción obtenida mediante el modelado estructural, este polimorfismo llevaría a un cambio en la estructura secundaria, lo que podría afectar la afinidad de los antifolatos con esta enzima y estar relacionado con los cambios en la sensibilidad del parásito a estos medicamentos (29). Este cambio estructural en la afinidad de los medicamentos con la enzima parasitaria, puede llevar a que los pacientes infectados con parásitos que tienen tal polimorfismo presenten menos mejoría o tengan más recaídas debido a una menor eficacia del tratamiento. Esta hipótesis debería ponerse a prueba en futuros estudios con ensayos farmacológicos adicionales y utilizando proteínas recombinantes normales y mutadas para ensayos in vitro que evalúen el efecto de los antifolatos sobre la actividad enzimática de cada una de ellas. 


\begin{tabular}{|c|c|}
\hline FA & GGACTGGTGAGAACGCGAGGGGTTCTAACGTGAATGAGATAGGGGTCGAGCCAGATAAA \\
\hline NR-1 & GGACTGGTGAGAACGCGAGGGGTTCTAACGTGAATGAGATAGGGGTCGAGCCĀ̄ATAAA \\
\hline Referencia & ACTGGTGAGAACGCGAGGGGTTCTAACGTGAATGAGATAGGGGTCGAGCCA $\overline{\mathrm{A}}$ ATAAA \\
\hline $\mathrm{RH}$ & GGACTGGTGAGAACGCGAGGGGTTCTAACGTGAATGAGATAGGGGTCGAGCCAGATAAA \\
\hline NR & 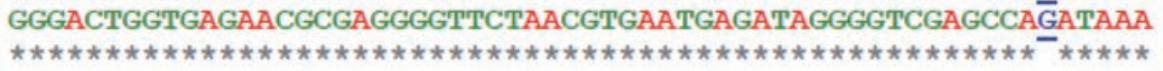 \\
\hline
\end{tabular}

Figura 5. Alineamiento de secuencias obtenidas para el exón 2 del gen dhps. FA: cepa del aislamiento colombiano 1 obtenida de paciente con HIV; NR: cepa del aislamiento colombiano 2 obtenida de paciente con HIV. Referencia: secuencia obtenida en Genbank de la cepa de referencia RH (cepa ATCC 50174), la cual se mantiene en el Laboratorio de Parasitología del Instituto Nacional de Salud. Los cambios encontrados en las secuencias obtenidas con respecto a la referencia se señalan en color azul y aparecen subrayados. El resto de la secuencia es idéntica para todas las muestras empleadas, como lo indican los asteriscos *** debajo del alineamiento.

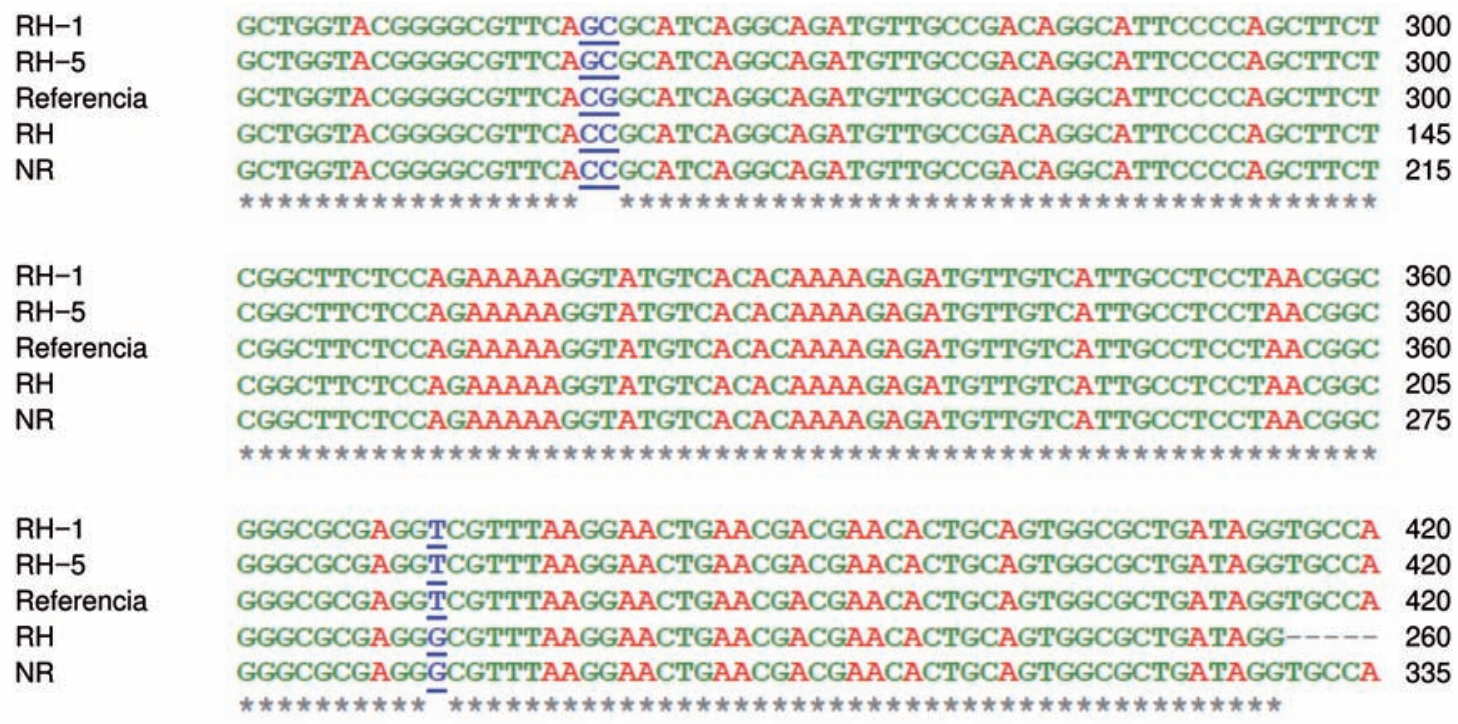

Figura 6. Alineamiento de secuencias obtenidas para el exón 4 del gen dhps. FA: cepa del aislamiento colombiano 1 obtenida de paciente con HIV; NR: cepa del aislamiento colombiano 2 obtenida de paciente con HIV. Referencia: secuencia obtenida en Genbank de la cepa de referencia RH (cepa ATCC 50174), la cual se mantiene en el Laboratorio de Parasitología del Instituto Nacional de Salud. Los cambios encontrados en las secuencias obtenidas con respecto a la referencia se señalan en color azul y aparecen subrayados. El resto de la secuencia es idéntica para todas las muestras empleadas, como lo indican los asteriscos *** debajo del alineamiento.

Entre las mutaciones génicas son más comunes las transiciones que las transversiones (30), aunque estas últimas pueden tener un mayor impacto en la funcionalidad de las proteínas en las que ocurre la mutación del ADN, impacto que puede ir desde cambios neutros hasta una funcionalidad ligeramente aumentada o disminuida, una pérdida de funcionalidad o un cambio de la funcionalidad de la proteína (enzima). Estos polimorfismos en posiciones análogas se han observado en organismos relacionados, incluido Plasmodium falciparum y Pneumocystis carinii, y se han asociado con resistencia a las sulfonamidas $(13,31)$.

En Colombia se hizo un estudio con el fin de determinar la frecuencia de mutaciones en los genes $\mathrm{dhfr}$ y dhps de $P$. falciparum asociadas con resistencia a la combinación de sulfadoxina y pirimetamina, en muestras de pacientes de tres zonas endémicas del país, y se encontró que en las poblaciones de $P$. falciparum analizadas prevalecían en los alelos asparagina 108, isoleucina 51 y glicina 437 , lo que indica un efecto acumulativo de mutaciones y la necesidad de vigilar la aparición de nuevos alelos mutantes que puedan conducir a la pérdida total de la eficacia de la combinación de sulfadoxina y pirimetamina (22).

Teniendo en cuenta que, al igual que $P$. falciparum, $T$. gondii, pertenece al rango apicomplexa y comparte con este muchas características, entre ellas la sensibilidad a los antifolatos, y que se han encontrado polimorfismos en los genes dhfr y dhps de cepas colombianas de $P$. falciparum, se resalta la importancia de la detección de estos polimorfismos en cepas circulantes en nuestro país. 

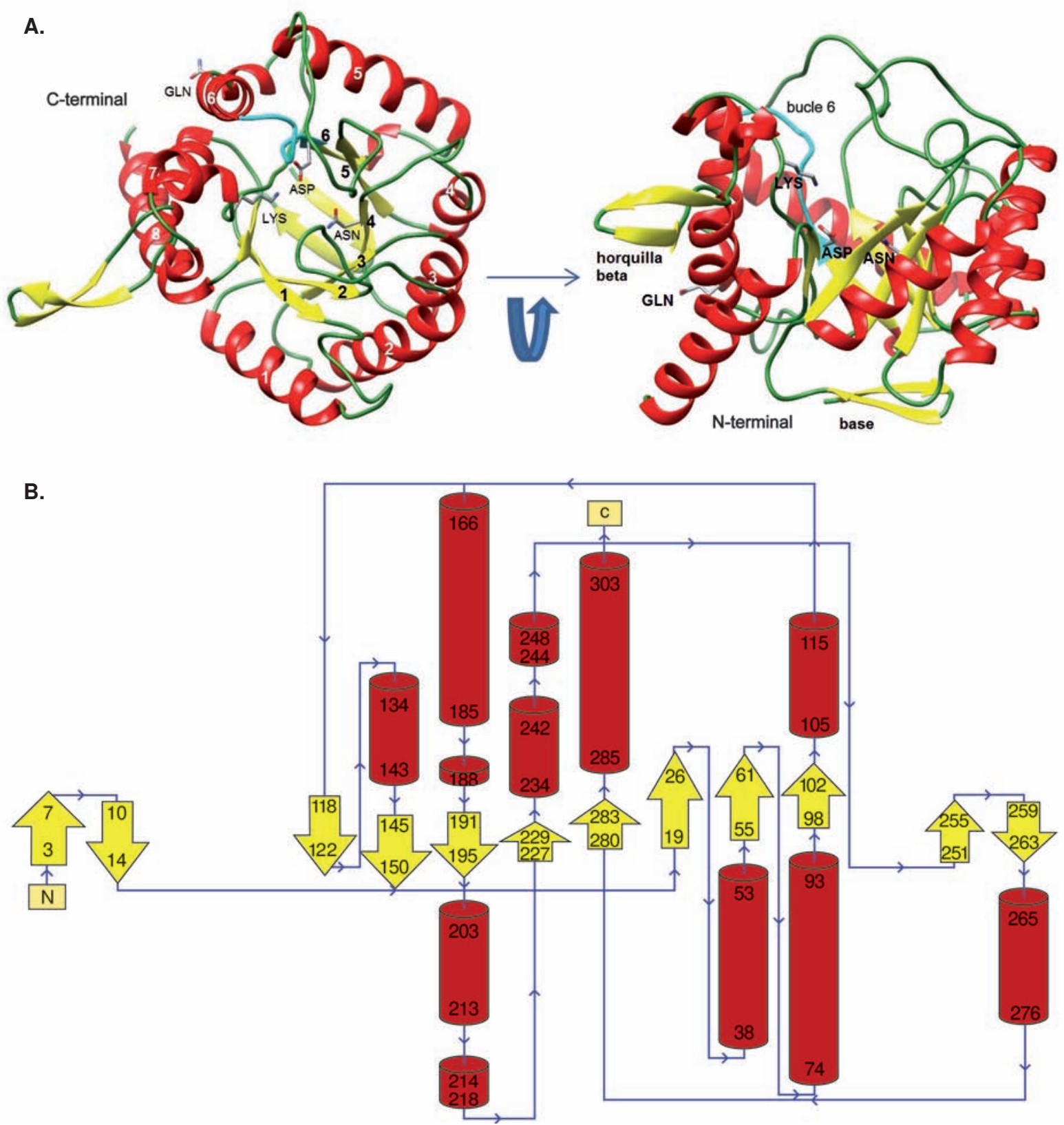

Figura 7. Modelo estructural de la enzima dihidropteroato- sintetasa (dhps) de Toxoplasma gondii. A) Estructura tridimensional de la enzima normal en la cual se muestran en rojo las hélices alfa (1-8) que se encuentran entre los aminoácidos 38-53, 74-93, 105$115,134-143,166-185,203-218,234-242,265-276$ y 285-303, y en amarillo, las láminas (1-8) del barril que se ubican entre los residuos 19-26, 55-61, 98-102, 118-122, 145-150, 191-195, 251-255 y 280-283. Además, se señalan los aminoácidos conservados en el bolsillo de unión a pterinas y, en azul magenta, el dominio de unión a PABA. B) Diagrama de la topología de las estructuras secundarias de la enzima

En Colombia existen reportes sobre la experiencia clínica y terapéutica en toxoplasmosis: en un estudio en pacientes con el HIV y con toxoplasmosis cerebral, se reveló una eficacia del tratamiento (sulfadoxina y pirimetamina más clindamicina) de $83 \%$, sin que se establecieran las causas del fracaso terapéutico en el $17 \%$ restante (10); asimismo, en un estudio sobre toxoplasmosis ocular se demostró una alta tasa de recaídas en pacientes tratados con diferentes esquemas de antiparasitarios (20).

Aunque no existen publicaciones científicas que describan la resistencia naturalmente adquirida de $T$. gondii a los antifolatos, en los estudios de Corea (12), Francia (6), Reino Unido (13) y Estados 
Unidos (14), se ha revelado la existencia de cepas con algún tipo de resistencia a pirimetamina y sulfadoxina (in vivo e in vitro). Estas variantes resistentes del parásito están relacionadas con la acumulación de mutaciones en el dominio de los genes que son blanco terapéutico de los antifolatos (dhfr y dhps). Por esta razón, es fundamental implementar metodologías que incluyan ensayos de inhibición (32) y medición de los niveles de resistencia de parásitos sensibles transformados in vitro con las diversas mutaciones del gen (33). Esto permitiría establecer un método de vigilancia para la aparición de mutantes resistentes de $T$. gondii.

Se recomienda la realización de futuros ensayos utilizando sistemas de expresión en organismos procariotas y eucariotas para producir proteínas recombinantes con mutaciones y sin ellas y, posteriormente, evaluar su efecto sobre la sensibilidad de dichas enzimas a cada uno de los antifolatos.

\section{Agradecimientos}

Los autores expresan su agradecimiento a todas las personas que colaboraron con el desarrollo de este trabajo en los Grupos de Parasitología y Fisiología Molecular del Instituto Nacional de Salud, el Departamento de Salud Pública de la Facultad de Medicina de la Universidad Nacional de Colombia y el Grupo de Parasitología Molecular de la Universidad del Quindío.

\section{Conflicto de intereses}

Los autores declaran que no existe ningún conflicto de intereses.

\section{Financiación}

Este trabajo fue financiado con recursos propios del Instituto Nacional de Salud, del Grupo de Parasitología Molecular de la Universidad del Quindío y del Departamento de Salud Pública de la Universidad Nacional de Colombia.

\section{Referencias}

1. Murat JB, Hidalgo HF, Brenier-Pinchart MP, Pelloux $\mathbf{H}$. Human toxoplasmosis: Which biological diagnostic tests are best suited to which clinical situations? Expert Rev Anti Infect Ther. 2013;11:943-56. http://dx.doi.org/10.1586/1478 7210.2013 .825441

2. Peña YR, Bernal R, Pavía N. Toxoplasmosis diseminada en niños. Informe de dos casos. Enfermedades Infecciosas y Microbiología. 2003:23:149-54.

3. Lindsay D, Blagburn B, Dubey J. Feline toxoplasmosis and the importance of the Toxoplasma gondii oocyst. Parasitology. 1997;19:448-61.
4. Wong SY, Remington JS. Biology of Toxoplasma gondii. AIDS. 1993;7:299-316. http://dx.doi.org/10.1097/00002030199303000-00001

5. Villena I, Aubert D, Leroux B, Dupouy D, Talmud M, Chemla C, et al. Pyrimethamine-sulfadoxine treatment of congenital toxoplasmosis: Follow-up of 78 cases between 1980 and 1997. Scand J Infect Dis. 1998;30:295-300. http:// dx.doi.org/10.1080/00365549850160963

6. Meneceur P, Bouldouyre M, Aubert D, Villena I, Menotti J, Sauvage V, et al. In vitro susceptibility of various genotypic strains of Toxoplasma gondii to pyrimethamine, sulfadiazine, and atovaquone antimicrobial agents and chemotherapy. Antimicrob Agents Chemother. 2008;52:1269-77. http://dx. doi.org/10.1128/AAC.01203-07

7. Lilue J, Müller UB, Steinfeldt T, Howard JC. Reciprocal virulence and resistance polymorphism in the relationship between Toxoplasma gondii and the house mouse. Elife. 2013;2:e01298. http://dx.doi.org/10.7554/eLife.01298

8. Gómez J, Castaño J, Montoya M, Loango N, López C, Sarmiento M, et al. Toxoplasmosis congénita en Colombia: análisis clínico y de laboratorio en 27 casos. Revista de Pediatría. 2000;35:52-7.

9. Gómez J. Tratamiento de la toxoplamosis: esquemas para la forma congénita y en el inmunosuprimido. Rev Salud Pública. 2002;4:35-42.

10. Gómez J, Alvarado F, Hernández C, Cuervo S, Saravia J. Tratamiento de la fase aguda de la toxoplasmosis cerebral con clindamicina-falcidar (pirimetamina-sulfadoxina) en pacientes infectados por VIH. Infectio. 2001;5:163-9.

11. Doliwa C, Xia D, Escotte-Binet S, Newsham EL, Sanya J S, Aubert D, et al. Identification of differentially expressed proteins in sulfadiazine resistant and sensitive strains of Toxoplasma gondii using difference-gel electrophoresis (DIGE). Int J Parasitol Drugs Drug Resist. 2013 5;3:35-44. http://dx.do/10.1016/j.ijpddr.2012.12.002.eCollection 2013

12. Eun J, Ho-Woo N. Changes in enzyme activity and expression of DHRF of Toxoplasma gondii by antifolates. Korean J Parasitol. 1998;6:191-8. http://dx.doi.org/10.3347/kjp. 1998.36.3.191

13. Aspinall TV, Joynson DH, Guy E, Hyde JE, Sims PF. The molecular basis of sulfonamide resistance in Toxoplasma gondii and implications for the clinical management of toxoplasmosis. J Infect Dis. 2002;185:1637-43. http://dx.doi. org/10.1086/340577

14. Nagamune K, Moreno S, Sibley D. Artemisinin-resistant mutants of Toxoplasma gondii have altered calcium homeostasis. Antimicrob Agents Chemother. 2007;51:381623. http://dx.doi.org/10.1128/AAC.00582-07

15. Katlama C, De Wit S, O'Doherty E, van Glabeke M, Clumeck N. Pyrimethamine-clindamycin Vs. pyrimethaminesulfadiazine as acute and long term therapy for toxoplasmic encephalitis in patients with AIDS. Clin Infect Dis. 1996;22:268-75. http://dx.doi.org/10.1093/clinids/22.2.268

16. Schmidt D, Hogh B, Hansen O, Dalhoff K, Petersen E. Treatment of infants with congenital toxoplasmosis: Tolerability and plasma concentrations of sulfadiazine and pyrimethamine. Eur J Pediatr. 2006;165:19-25. http://dx.doi. org/10.1007/s00431-005-1665-4 
17. Doliwa C, Escotte-Binet S, Aubert D, Sauvage V, Velard F, Schmid A, et al. Sulfadiazine resistance in Toxoplasma gondii: No involvement of overexpression or polymorphisms in genes of therapeutic targets and $A B C$ transporters. Parasite. 2013;20:19. http://dx.doi.org/10. 1051/parasite/2013020

18. Donald RGK, Roos DS. Stable molecular transformation of Toxoplasma gondii: A selectable dihydrofolate reductase-thymidylate synthase marker based on drugresistance mutations in malaria. Proc Natl Acad Sci USA. 1993;90:11703-7. http://dx.doi.org/10.1073/pnas.90. 24.11703

19. Reynolds MG, Roos DS. A biochemical and genetic model for parasite resistance to antifolates. Toxoplasma gondii provides insights into pyrimethamine and cycloguanil resistance in Plasmodium falciparum. J Biol Chem. 1998; 273:3461-9 http://dx.doi.org/10.1074/jbc.273.6.3461

20. Martín-Hernández I, García-Izquierdo S. Toxoplasmosis: infección oportunista en pacientes con el síndrome de inmunodeficiencia adquirida. Rev Biomed. 2003;14:101-11.

21. de la Torre A, Ríos-Cadavid AC, Cardozo-García CM, Gómez-Marín JE. Frequency and factors associated with recurrences of ocular toxoplasmosis in a referral centre in Colombia. Br J Ophthalmol. 2009;93:1001-4. http://dx.doi. org/10.1136/bjo.2008.155861

22. Galindo J, Cristiano J, Knudson A, Nicholls RS, Guerra A. Mutaciones puntuales en los genes dhfr y dhps de Plasmodium falciparum de tres regiones endémicas para malaria en Colombia. Biomédica. 2010;30:56-64. http://dx. doi.org/10.7705/biomedica.v33i1.643

23. Robles S, Hortúa A, Zimmermann B. Cloning and preliminary characterization of the dihydroorotase from Toxoplasma gondii. Mol Biochem Parasitol. 2006;148:93-8. http://dx.doi.org/10.1016/j.molbiopara.2006.03.003

24. Mejías I, Zimmermann B. Molecular cloning, recombinant expression, and partial characterization of the aspartate transcarbamoylase from Toxoplasma gondii. Mol Biochem Parasitol. 2002;119:191-201. http://dx.doi.org/10.1016/s01666851(01)00415-7

25. Cortés LJ, Arévalo A, Sofía D. Aislamiento de Toxoplasma gondii a partir de líquido cefalorraquídeo de dos pacientes
VIH positivos. Infectio. 2013;17:61-5. http://dx.doi.org/10. 1016/s0123-9392(13)70164-4

26. Wang L, Cheng HW, Huang KQ, Xu YH, Li YN, Du J, et al. Toxoplasma gondii prevalence in food animals and rodents in different regions of China: Isolation, genotyping and mouse pathogenicity. Parasit Vectors. 2013;6:273. http://dx. doi.org/10.1186/1756-3305-6-273

27. Wang L, Chen H, Liu D, Huo X, Gao J, Song X, et al. Genotypes and mouse kit virulence of Toxoplasma gondii isolates from animals and humans in China. PLoS One. 2013;6:e53483. http://dx.doi.org/10.1371/journal.pone. 0053483

28. Ponce N, Gómez J. Estandarización y validación clínica de la prueba de reacción en cadena de la polimerasa (PCR) para diagnóstico de toxoplasmosis cerebral en pacientes infectados por el VIH. Infectio. 2003;7:8-14.

29. Chaudary K, Fox B, Bzik D. Comparative aspects of nucleotide and aminoacid metabolism in Toxoplasma gondii and other apicomplexa. In: Louis M, Kami K, editors. Toxoplasma gondii: The model apicomplexan: Perspectives and methods. First edition. Amsterdam: Elsevier Ltda; 2007. p. 465-504. http://dx.doi.org/10.1016/b978-0-12-396481-6. 00020-9

30. Wakeley J. The excess of transitions among nucleotide substitutions: New methods of estimating transition bias underscore its significance. Trends Ecol Evol. 1996;11:15862. http://dx.doi.org/10.1016/0169-5347(96)10009-4

31. Escalante AA, Lal AA, Ayala FJ. Genetic polymorphism and natural selection in the malaria parasite Plasmodium falciparum. Genetics. 1998;149:189-202. http://dx.doi.org/10. 1186/1475-2875-11-60

32. Triglia T, Menting JG, Wilson C, Cowman AF. Mutations in dihydropteroate synthase are responsible for sulfone and sulfonamide resistance in Plasmodium falciparum. Proc Natl Acad Sci USA. 1997;94:13944-9. http://dx.doi.org/10.1073/ pnas.94.25.13944

33. Triglia T, Wang P, Sims PF, Hyde JE, Cowman AF. Allelic exchange at the endogenous genomic locus in Plasmodium falciparum proves the role of dihydropteroate synthase in sulfadoxine-resistant malaria. EMBO J. 1998;17:3807-15. http://dx.doi.org/10.1093/emboj/17.14.3807 